\title{
ON THE PRODUCT OF A RIESZ SET AND A SMALL $p$ SET
}

\author{
HIROSHI YAMAGUCHI
}

\begin{abstract}
ABSTRACr. Let $Z^{+}$be the semigroup consisting of all nonnegative integers. By a famous theorem of Bochner, $Z^{+} \times Z^{+}$is a Riesz set in $Z \oplus Z$. In this paper, we prove that the product set of a Riesz set and a small $p$ set is a small $p$ set.
\end{abstract}

1. Introduction. Let $T$ be the circle group and $Z^{+}$the semigroup consisting of nonnegative integers. Then, by the famous F. and M. Riesz theorem, each measure on $T$ whose Fourier-Stieltjes transform vanishes off $Z^{+}$is absolutely continuous with respect to the Lebesgue measure on $T$. Moreover, by a well-known theorem of Bochner, each measure on $T^{2}$ whose Fourier-Stieltjes transform vanishes off $Z^{+} \times Z^{+}$is absolutely continuous with respect to the Lebesgue measure on $T^{2}$.

In this paper, we prove that the product set of a Riesz set and a small $p$ set is a small $p$ set. We use Glicksberg's ideas [1] and the theory of disintegration.

For a LCA group $G, C_{c}(G), C_{0}(G), L^{1}(G)$ and $M(G)$ denote the usual spaces. For a subset $E$ of $\hat{G}, M_{E}(G)$ denotes the space consisting of all measures in $M(G)$ whose Fourier-Stieltjes transforms vanish off $E$. We denote the Haar measure on $G$ by $m_{G}$.

Definition. Let $G$ be a LCA group. For a positive integer $p$, a closed subset $E$ of $\hat{G}$ is called a small $p$ set if the following is satisfied:

For each $\mu \in M_{E}(G), \mu^{p}(=\mu * \mu * \cdots * \mu$ ( $p$ times $)$ ) belongs to $L^{1}(G)$. In particular, a small 1 set is called a Riesz set.

LEMma 1 [4]. Let $p$ be a positive integer. Then we have

$$
t_{1} t_{2} \ldots t_{p}=\sum_{i=1}^{l_{p}} A_{i} \Phi_{i}\left(t_{1}, t_{2}, \ldots, t_{p}\right)^{p} \text { for each }\left(t_{1}, t_{2}, \ldots, t_{p}\right) \in C^{p}
$$

where $A_{i} \in C$ (complex numbers) and $\Phi_{i}$ are linear forms of $t_{1}, t_{2}, \ldots, t_{p}$.

\section{Main theorem.}

TheOREM 1. Let $G_{1}$ and $G_{2}$ be metrizable $\sigma$-compact LCA groups. Let $E_{1}$ be a small $p$ set in $\hat{G}_{1}$ and $E_{2}$ a Riesz set in $\hat{G}_{2}$. Then $E_{1} \times E_{2}$ is a small $p$ set in $\widehat{G}_{1} \oplus G_{2}$.

Proof. Let $\mu$ be a measure in $M_{E_{1} \times E_{2}}\left(G_{1} \oplus G_{2}\right)$. Let $\pi$ be the projection from $G_{1} \oplus G_{2}$ onto $G_{2}$. Put $\eta=\pi(|\mu|)$ (continuous image under $\pi$ ). Then, by disintegration theory, there exists a family $\left\{\lambda_{h}\right\}_{h \in G_{2}}$ in $M\left(G_{1} \oplus G_{2}\right)$ with the following properties:

Received by the editors November 27, 1979.

AMS (MOS) subject classifications (1970). Primary 43A05, 43A25.

(C) 1981 American Mathematical Society 0002-9939/81/0000-0075/\$02.50 
(1) $h \mapsto \lambda_{h}(f)$ is a Borel measurable function of $h$ for each bounded Borel measurable function $f$ on $G_{1} \oplus G_{2}$,

(2) $\operatorname{supp}\left(\lambda_{h}\right) \subset \pi^{-1}(\{h\})=G_{1} \times\{h\}$,

(3) $\left\|\lambda_{h}\right\|<1$,

(4) $\mu(g)=\int_{G_{2}} \lambda_{h}(g) d \eta(h)$ for each bounded Borel measurable function $g$ on $G_{1} \oplus G_{2}$.

By (2), we have $d \lambda_{h}(x, y)=d \nu_{h}(x) \times d \delta_{h}(y)$, where $\nu_{h} \in M\left(G_{1}\right)$ and $\delta_{h}$ is the Dirac measure at $h$. By the method used in [1, pp. 425-426], we have $\nu_{h} \in M_{E_{1}}\left(G_{1}\right)$ a.a. $h(\eta)$.

That is, there exists a Borel measurable set $K$ in $G_{2}$ with $\eta\left(G_{2} \backslash K\right)=0$ such that $\nu_{h} \in M_{E_{1}}\left(G_{1}\right)$ for $h \in K$.

Claim 1. $\eta$ belongs to $L^{1}\left(G_{2}\right)$.

For $\gamma_{0}=\left(\gamma_{1}, \gamma_{2}\right) \in \widehat{G_{1} \oplus G_{2}}$, we have $\pi\left(\gamma_{0} \mu\right)^{\wedge}(\gamma)=\hat{\mu}\left(-\gamma_{1}, \gamma-\gamma_{2}\right)$. Hence $\pi\left(\gamma_{0} \mu\right)$ belongs to $M_{\gamma_{2}+E_{2}}\left(G_{2}\right)$. Since $E_{2}$ is a Riesz set, $\gamma_{2}+E_{2}$ is also a Riesz set. Hence $\pi\left(\gamma_{0} \mu\right)$ belongs to $L^{1}\left(G_{2}\right)$. On the other hand, there exists a sequence $\left\{p_{n}\right\}$ in $\operatorname{Trig}\left(G_{1} \oplus G_{2}\right)$ such that $\lim _{n \rightarrow \infty}\left\|p_{n} \mu-|\mu|\right\|=0$. Hence we have

$$
\lim _{n \rightarrow \infty}\left\|\pi\left(p_{n} \mu\right)-\pi(|\mu|)\right\|=0 \text {. }
$$

Thus Claim 1 is proved.

Claim 2. $\left(h_{1}, \ldots, h_{p}\right) \mapsto \lambda_{h_{1}} * \cdots * \lambda_{h_{p}}(g)=\left(\nu_{h_{1}} * \cdots * \nu_{h_{p}}\right) \times$ $\delta_{\left(h_{1}+\cdots+h_{p}\right)}(g)$ is a Borel measurable function on $G_{2}^{p}$ for each bounded Borel function $g$ on $G_{1} \oplus G_{2}$.

Let $f_{j} \in C_{c}\left(G_{1} \oplus G_{2}\right)$ and put $f\left(x_{1}, \ldots, x_{p}\right)=f_{1}\left(x_{1}\right) \ldots f_{p}\left(x_{p}\right)$ for $\left(x_{1}, \ldots, x_{p}\right)$ $\in\left(G_{1} \oplus G_{2}\right)^{p}$. Then $\left(\lambda_{h_{1}} \times \cdots \times \lambda_{h_{p}}\right)(f)=\lambda_{h_{1}}\left(f_{1}\right) \ldots \lambda_{h_{p}}\left(f_{p}\right)$ so that by (1)

(5) $\left(h_{1}, \ldots, h_{p}\right) \mapsto\left(\lambda_{h_{1}} \times \cdots \times \lambda_{h_{p}}\right)(f)$ is Borel measurable.

Since $\left\{\sum_{1}^{n} f_{1 i}\left(x_{1}\right) \ldots f_{p i}\left(x_{p}\right) ; f_{j i} \in C_{c}\left(G_{1} \oplus G_{2}\right)\right\}$ is dense in $C_{0}\left(\left(G_{1} \oplus G_{2}\right)^{p}\right),(5)$ is Borel measurable for $f \in C_{0}\left(\left(G_{1} \oplus G_{2}\right)^{p}\right)$, hence, also for bounded Borel functions $f$ on $\left(G_{1} \oplus G_{2}\right)^{p}$. Let $\pi_{p}\left(x_{1}, \ldots, x_{p}\right)=x_{1}+\cdots+x_{p}$ for $\left(x_{1}, \ldots, x_{p}\right) \in$ $\left(G_{1} \oplus G_{2}\right)^{p}$. Then for bounded Borel $g$ on $G_{1} \oplus G_{2}$ we have $\lambda_{h_{1}} * \cdots * \lambda_{h_{p}}(g)=$ $\left(\lambda_{h_{1}} \times \cdots \times \lambda_{h_{p}}\right)\left(g \circ \pi_{p}\right)$ and the claim follows.

Hence, by Claim 2, we can define a measure $\xi$ in $M\left(G_{1} \oplus G_{2}\right)$ as follows

$$
\begin{aligned}
& \xi(f)=\overbrace{\int_{G_{2}} \int_{G_{2}} \cdots \int_{G_{2}}}\left\{\left(\nu_{h_{1}} * \nu_{h_{2}} * \cdots * \nu_{h_{p}}\right) \times \delta_{\left(h_{1}+h_{2}+\cdots+h_{p}\right)}\right\} \\
& \times(f) d \eta\left(h_{1}\right) d \eta\left(h_{2}\right) \ldots d \eta\left(h_{p}\right)
\end{aligned}
$$

for $f \in C_{0}\left(G_{1} \oplus G_{2}\right)$.

Claim 3. $\xi=\mu^{p}$.

Let $\left(\gamma_{1}, \gamma_{2}\right)$ be in $\widehat{G_{1} \oplus G_{2}}$. Then we have

Thus we have $\xi=\mu^{p}$.

$$
\begin{aligned}
\hat{\xi}\left(\gamma_{1}, \gamma_{2}\right) & =\prod_{i=1}^{p} \int_{G_{2}} \hat{\nu}_{h_{i}}\left(\gamma_{1}\right)\left(-h_{i}, \gamma_{2}\right) d \eta\left(h_{i}\right) \\
& =\left\{\hat{\mu}\left(\gamma_{1}, \gamma_{2}\right)\right\}^{p}=\left(\mu^{p}\right)^{\wedge}\left(\gamma_{1}, \gamma_{2}\right) .
\end{aligned}
$$

Let $E$ be a Borel measurable set in $G_{1} \oplus G_{2}$ with $m_{G_{1} \oplus G_{2}}(E)=0$, where $m_{G_{1} \oplus G_{2}}$ 
denotes the Haar measure on $G_{1} \oplus G_{2}$. Then there exists a Borel measurable set $F_{2}$ in $G_{2}$ such that (i) $m_{G_{2}}\left(F_{2}\right)=0$ and (ii) $m_{G_{1}}\left(E_{y}\right)=0$ if $y \notin F_{2}$, where $E_{y}=\{x \in$ $\left.G_{1} ;(x, y) \in E\right\}$. Let $\alpha_{p}$ be the homomorphism from $G_{2}^{p}$ onto $G_{2}$ such that $\alpha_{p}\left(h_{1}, h_{2}, \ldots, h_{p}\right)=h_{1}+h_{2}+\cdots+h_{p}$. We note that $\nu_{h_{1}} * \nu_{h_{2}} * \cdots * \nu_{h_{p}}$ belongs to $L^{1}\left(G_{1}\right)$ for $\left(h_{1}, h_{2}, \ldots, h_{p}\right) \in K^{p}$ by Lemma 1 and

$$
\eta^{p}\left(F_{2}\right)=(\widetilde{\eta \times \eta \times \cdots \times \eta})\left(\alpha_{p}^{-1}\left(F_{2}\right)\right) \text {. }
$$

Hence, by Claim 1 and Claim 3, we have

$$
\begin{aligned}
\mu^{p}(E)= & \int_{G_{2}} \int_{G_{2}} \cdots \int_{G_{2}}\left\{\left(\nu_{h_{1}} * \nu_{h_{2}} * \cdots * \nu_{h_{p}}\right) \times \delta_{\left.\left(h_{1}+h_{2}+\cdots+h_{p}\right)\right\}}\right. \\
& \times\left(\chi_{E}\right) d \eta\left(h_{1}\right) d \eta\left(h_{2}\right) \ldots d \eta\left(h_{p}\right) \\
= & \int_{K^{p}}\{"\} d(\eta \times \eta \times \cdots \times \eta)\left(h_{1}, h_{2}, \ldots, h_{p}\right) \\
= & \int_{K^{p} \cap \alpha_{p}^{-1}\left(F_{2}\right)}\{"\} d(\eta \times \eta \times \cdots \times \eta)\left(h_{1}, h_{2}, \ldots, h_{p}\right) \\
& +\int_{K^{p} \backslash \alpha_{p}^{-1}\left(F_{2}\right)}\{"\} d(\eta \times \eta \times \cdots \times \eta)\left(h_{1}, h_{2}, \ldots, h_{p}\right)=0 .
\end{aligned}
$$

Hence we have $\mu^{p} \in L^{1}\left(G_{1} \oplus G_{2}\right)$. Thus $E_{1} \times E_{2}$ is a small $p$ set in $\widehat{G_{1} \oplus G_{2}}$. Q.E.D.

Dr. S. Saeki kindly pointed out the following Lemma 2 to the author.

LEMMA 2. Suppose the product set $E_{1} \times E_{2}$ of a small $p$ set $E_{1}$ in $\hat{G}_{1}$ and a Riesz set $E_{2}$ in $\hat{G}_{2}$ is a small $p$ set in $\hat{G}_{1} \oplus \hat{G}_{2}$ for all metrizable $L C A$ groups $G_{1}$ and $G_{2}$. Then the product set $E_{3} \times E_{4}$ of a small $p$ set $E_{3}$ in $\hat{G}_{3}$ and a Riesz set $E_{4}$ in $\hat{G}_{4}$ is a small $p$ set in $G_{3} \oplus G_{4}$ for all LCA groups $G_{3}$ and $G_{4}$.

Proof. Suppose there exists a measure $\mu$ in $M_{E_{3} \times E_{4}}\left(G_{3} \oplus G_{4}\right)$ such that $\mu^{p}$ does not belong to $L^{1}\left(G_{3} \oplus G_{4}\right)$. Then, by Corollary 3 of [2], there exists a measure $\sigma$ in $M_{o}\left(G_{3} \oplus G_{4}\right)$ such that

$$
(\mu * \sigma)^{p}=\mu^{p} * \sigma^{p} \notin L^{1}\left(G_{3} \oplus G_{4}\right) .
$$

Since $\sigma \in M_{0}\left(G_{3} \oplus G_{4}\right)$, there exist open $\sigma$-compact subgroups $\Gamma_{i}$ of $\hat{G}_{i}$ such that $\operatorname{supp}(\hat{\sigma}) \subset \Gamma_{3} \times \Gamma_{4}(i=3,4)$. On the other hand, $\hat{\Gamma}_{3}$ and $\hat{\Gamma}_{4}$ are metrizable LCA groups. Evidently $E_{3} \cap \Gamma_{3}$ is a small $p$ set in $\Gamma_{3}$ and $E_{4} \cap \Gamma_{4}$ is a Riesz set in $\Gamma_{4}$. Hence, by the hypothesis of this Lemma, $\left(E_{3} \cap \Gamma_{3}\right) \times\left(E_{4} \cap \Gamma_{4}\right)$ is a small $p$ set in $\Gamma_{3} \oplus \Gamma_{4}$. Therefore, since $\sigma * \mu \in M_{\left(E_{3} \cap \Gamma_{3}\right) \times\left(E_{4} \cap \Gamma_{4}\right)}\left(G_{3} \oplus G_{4}\right)$, we have $(\sigma * \mu)^{p} \in$ $L^{1}\left(G_{3} \oplus G_{4}\right)$.

This contradicts (A). Q.E.D.

LEMMA 3. Let $G_{3}$ and $G_{4}$ be metrizable LCA groups. Let $E_{3}$ be a small $p$ set in $\hat{G}_{3}$ and $E_{4} a$ Riesz set in $\hat{G}_{4}$. Then $E_{3} \times E_{4}$ is a small $p$ set in $\hat{G}_{3} \oplus G_{4}$.

Proof. Let $\mu$ be a measure in $M_{E_{3} \times E_{4}}\left(G_{3} \oplus G_{4}\right)$. Then there exist open metrizable $\sigma$-compact subgroups $G_{1} \subset G_{3}$ and $G_{2} \subset G_{4}$ such that $\operatorname{supp}(\mu)$ is contained in $G_{1} \oplus G_{2}$. Let $\pi_{G_{2}}$ be the projection from $G_{1} \oplus G_{2}$ onto $G_{2}$. Put $\eta^{\prime}=\pi_{G_{2}}(|\mu|)$. Then, 
by disintegration theory, there exists a family $\left\{\lambda_{h}^{\prime}\right\}_{h \in G_{2}}$ in $M\left(G_{1} \oplus G_{2}\right)$ such that

(1) $h \mapsto \lambda_{h}^{\prime}(f)$ is a Borel measurable function of $h$ for each bounded Borel measurable function $f$ on $G_{1} \oplus G_{2}$,

(2) $\operatorname{supp}\left(\lambda_{h}^{\prime}\right) \subset G_{1} \times\{h\}$,

(3) $\left\|\lambda_{h}^{\prime}\right\|<1$ and

(4) $\mu(g)=\int_{G_{2}} \lambda_{h}^{\prime}(g) d \eta^{\prime}(h)$ for each bounded Borel measurable function $g$ on $G_{1} \oplus G_{2}$.

Since $G_{1} \oplus G_{2}$ is $\sigma$-compact and metrizable, there exists a countable dense set $\mathbb{Q}=\left\{f_{m}\right\}$ in $C_{0}\left(G_{1} \oplus G_{2}\right)$. For each $g \in C_{0}\left(G_{1} \oplus G_{2}\right)$, we define a function $\tilde{g}$ in $C_{0}\left(G_{3} \oplus G_{4}\right)$ by $\tilde{g}(x)=g(x)$ for $x \in G_{1} \oplus G_{2}$ and $\tilde{g}(x)=0$ for $x \notin G_{1} \oplus G_{2}$. We define measures $\eta \in M\left(G_{4}\right)$ and $\lambda_{h} \in M\left(G_{3} \oplus G_{4}\right)\left(h \in G_{4}\right)$ as follows

$$
\begin{aligned}
\eta(F) & =\eta^{\prime}\left(G_{2} \cap F\right) \text { for a Borel measurable set } F \text { in } G_{4}, \\
\lambda_{h}\left(F^{\prime}\right) & = \begin{cases}\lambda_{h}^{\prime}\left(F^{\prime} \cap G_{1} \oplus G_{2}\right) & \text { if } h \in G_{2}, \\
0 & \text { if } h \in G_{4} \backslash G_{2},\end{cases}
\end{aligned}
$$

for a Borel measurable set $F^{\prime}$ in $G_{3} \oplus G_{4}$. Then we have the following

(6) $h \mapsto \lambda_{h}(f)$ is a Borel measurable function of $h$ for each bounded Borel measurable function $f$ on $G_{3} \oplus G_{4}$,

(7) $\operatorname{supp}\left(\lambda_{h}\right) \subset G_{1} \times\{h\} \subset G_{3} \times\{h\}$,

(8) $\left\|\lambda_{h}\right\|<1$,

(9) $\mu(g)=\int_{G_{4}} \lambda_{h}(g) d \eta(h)$ for each bounded Borel measurable function $g$ on $G_{3} \oplus G_{4}$.

From (7), we have $d \lambda_{h}(x, y)=d \nu_{h}(x) \times d \delta_{h}(y)$, where $\nu_{h}$ is a measure in $M\left(G_{3}\right)$ with $\operatorname{supp}\left(\nu_{h}\right) \subset G_{1}$. Noting that $\operatorname{supp}(\eta) \subset G_{2}$, we may apply Lusin's theorem and regularity of $\eta$ to obtain for each positive integer $n$ a compact subset $K_{n}$ of $\operatorname{supp}(\eta)$ such that

(i) $\eta\left(G_{2} \backslash K_{n}\right)<1 / n$,

(ii) $h \mapsto \lambda_{h}\left(\tilde{f}_{m}\right)$ is a continuous function on $K_{n}$ for each $\tilde{f}_{m} \in \tilde{\mathbb{Q}}=\{\tilde{f} ; f \in \mathbb{Q}\}$, and

(iii) for each $x \in K_{n}$ and neighborhood $V$ of $x, \eta\left(V \cap K_{n}\right)>0$.

Since $\tilde{\mathscr{Q}}$ is dense in $C_{0}\left(G_{1} \oplus G_{2}\right)^{\sim}=\left\{\tilde{f} ; f \in C_{0}\left(G_{1} \oplus G_{2}\right)\right\}$ we may replace (ii) by

(ii) $h \mapsto \lambda_{h}(\tilde{f})$ is continuous on $K_{n}$ for each $\tilde{f} \in C_{0}\left(G_{1} \oplus G_{2}\right)^{2}$.

Claim 1. $\nu_{h} \in M_{E_{3}}\left(G_{3}\right)$ for $h \in K_{n}$.

Let $f \in L^{1}\left(\hat{G}_{3}\right)$ with $\operatorname{supp}(f) \subset E_{3}^{c}$. Then since $\hat{\mu}\left(\gamma_{1}, \gamma_{2}\right)=0$ for $\gamma_{1} \notin E_{3}$ we have

$$
\begin{aligned}
0 & =\int_{\hat{G}_{3}} \hat{\mu}\left(\gamma_{1}, \gamma_{2}\right) f\left(\gamma_{1}\right) d \gamma_{1} \\
& =\int_{\hat{G}_{3}} \int_{G_{4}} \int_{G_{3}}\left(-x, \gamma_{1}\right) d \nu_{h}(x)\left(-h, \gamma_{2}\right) d \eta(h) f\left(\gamma_{1}\right) d \gamma_{1} \\
& =\int_{G_{4}} \int_{G_{3}} \hat{f}(x) d \nu_{h}(x)\left(-h, \gamma_{2}\right) d \eta(h) \\
& =\int_{G_{4}} \nu_{h}(\hat{f})\left(-h, \gamma_{2}\right) d \eta(h) .
\end{aligned}
$$


Hence, for each $F \in L^{1}\left(\hat{G}_{4}\right)$ and $f \in L^{1}\left(\hat{G}_{3}\right)$ with $\operatorname{supp}(f) \subset E_{3}^{c}$ we have

$$
\begin{aligned}
0 & =\int_{\hat{G}_{4}} \int_{G_{4}} \nu_{h}(\hat{f})\left(-h, \gamma_{2}\right) d \eta(h) F\left(\gamma_{2}\right) d \gamma_{2} \\
& =\int_{G_{4}} \nu_{h}(\hat{f}) \hat{F}(h) d \eta(h) .
\end{aligned}
$$

Since $L^{1}\left(\hat{G}_{4}\right)^{\wedge}$ is dense in $C_{0}\left(G_{4}\right)$, hence also in $L^{1}(\eta)$, (11) holds for all $F \in L^{1}(\eta)$. It follows from (6) that $h \mapsto \nu_{h}(\hat{f})$ is bounded and Borel measurable, hence in $L^{\infty}(\eta)$, and so by (11)

$$
\nu_{h}(\hat{f})=0, \quad \eta \text {-a.e. }
$$

Let $\beta \in C_{c}\left(G_{4}\right)$ satisfy $\beta=1$ on $K_{n}, \beta=0$ off $G_{2}$, and set $g(x, y)=$ $\left(f * m_{G_{1}^{\perp}}\right)^{\wedge}(x) \beta(y)$, where $G_{1}^{\perp}$ is the annihilator of $G_{1}$. Then $g \in C_{0}\left(G_{1} \oplus G_{2}\right)^{\sim}$ and, since $\operatorname{supp}\left(\nu_{h}\right) \subset G_{1}$, we have $\lambda_{h}(g)=\nu_{h}\left(\left(f * m_{G_{1}^{1}}\right)^{\wedge}\right) \delta_{h}(\beta)=\nu_{h}(\hat{f})$ for each $h \in$ $K_{n}$. Hence, $h \mapsto \nu_{h}(\hat{f})$ is continuous on $K_{n}$ by (ii)', and this together with (12) and (iii) shows that $\nu_{h}(\hat{f})=0$ for each $h \in K_{n}$. Thus, for $h \in K_{n}, 0=\nu_{h}(\hat{f})=$ $\int_{\hat{G}_{3}} \hat{\nu}_{h}\left(\gamma_{1}\right) f\left(\gamma_{1}\right) d \gamma_{1}$.

Since $f$ is any function in $L^{1}\left(\hat{G}_{3}\right)$ with $\operatorname{supp}(f) \subset E_{3}^{c}$ we have $\hat{\nu}_{h}\left(\gamma_{1}\right)=0$ on $E_{3}^{c}$, and the claim is established. Moreover, since $\eta\left(G_{4} \backslash \cup{ }_{1}^{\infty} K_{n}\right)=0$ we have proved that $\nu_{h} \in M_{E_{3}}\left(G_{3}\right)$ a.a. $h(\eta)$.

Since $E$ is a Riesz set, we may prove that $\eta \in L^{1}\left(G_{4}\right)$ by arguing as in Theorem 1.

Claim 2. $\left(h_{1}, h_{2}, \ldots, h_{p}\right) \mapsto\left\{\left(\nu_{h_{1}} * \nu_{h_{2}} * \cdots * \nu_{h_{3}}\right) \times \delta_{\left(h_{1}+h_{2}+\cdots+h_{3}\right)}\right\}(g)$ is a Borel measurable function on $\left(G_{4}\right)^{p}$ for each $g \in C_{0}\left(G_{3} \oplus G_{4}\right)$.

Indeed, since $\nu_{h}=0$ if $h \notin G_{2}$, we have $\left(\nu_{h_{1}} * \nu_{h_{2}} * \cdots * \nu_{h^{\prime}}\right) \times \delta_{\left(h_{1}+h_{2}+\cdots+h_{p}\right)}$ $=0$ for $\left(h_{1}, h_{2}, \ldots, h_{p}\right) \notin\left(G_{2}\right)^{p}$.

On the other hand, $\lambda_{n}=\nu_{h} \times \delta_{h}$ may be regarded as a measure in $M\left(G_{1} \oplus G_{2}\right)$.

Since $G_{1}$ and $G_{2}$ are $\sigma$-compact metrizable LCA groups, we may prove Claim 2 by arguing as in the proof of Claim 2, Theorem 1 .

We now define a measure $\xi$ in $M\left(G_{3} \oplus G_{4}\right)$ as follows

$$
\xi(f)=\int_{G_{4}} \cdots \int_{G_{4}}\left\{\left(\nu_{h_{1}} * \cdots * \nu_{h_{p}}\right) \times \delta_{\left(h_{1}+\cdots+h_{p}\right)}\right\}(f) d \eta\left(h_{1}\right) \ldots d \eta\left(h_{p}\right)
$$

for $f \in C_{0}\left(G_{3} \oplus G_{4}\right)$.

Then we have $\xi=\mu^{p}$. Let $E_{0}$ be a Borel measurable set in $G_{3} \oplus G_{4}$ with $m_{G_{3} \oplus G_{4}}\left(E_{0}\right)=0$. Put $E=E_{0} \cap G_{1} \oplus G_{2}$. Since $\operatorname{supp}\left(\mu^{p}\right) \subset G_{1} \oplus G_{2}$, we have $\mu^{P}\left(E_{0}\right)=\mu^{P}(E)$. Moreover, $m_{G_{1} \oplus G_{2}}(E)=0$. Thus we can prove that $\mu^{p}\left(E_{0}\right)=0$ by using the techniques employed in Theorem 1. That is $\mu^{p} \in L^{1}\left(G_{3} \oplus G_{4}\right)$. This completes the proof.

From Lemma 2 and Lemma 3, we obtain the following main theorem.

THEOREM 2. Let $G_{1}$ and $G_{2}$ be LCA groups. Let $E_{1}$ be a small p set in $\hat{G}_{1}$ and $E_{2} a$ Riesz set in $\hat{G}_{2}$. Then $E_{1} \times E_{2}$ is a small $p$ set in $\widehat{G}_{1} \oplus G_{2}$. 
Finally, the author wishes to express his thanks to Dr. S. Saeki for his valuable advice.

\section{REFERENCES}

1. I. Glicksberg, Fourier-Stieltjes transforms with small supports, Illinois J. Math. 9 (1965), 418-426.

2. L. Pigno and S. Saeki, Fourier-Stieltjes transforms which vanish at infinity, Math. Z. 141 (1975), 83-91.

3. W. Rudin, Fourier analysis on groups, Interscience, New York, 1962.

4. H. Yamaguchi, Remarks on Riesz sets, Hokkaido Math. J. 7 (1978), 328-335.

Department of Mathematics, Josai University, SAKado, SATtama, JaPAN 